\title{
Sleep disorders and gene NPAS2 polymorphism in male population in Russia/Siberia: MONICA-psychosocial study
}

\author{
V. Gafarov ${ }^{1,2 *}$, I. Gagulin ${ }^{1,2}$, A. Gafarova ${ }^{1,2}$, D. Panov ${ }^{1,2}$, E. Krymov ${ }^{1,2}$, \\ M. Voevoda ${ }^{1,2}$, V. Maximov ${ }^{1,2}$, E. Gromova ${ }^{1,2}$ \\ ${ }^{1}$ NIITPM - Branch of Institute of Cytology and Genetics SB RAS, Novosibirsk, Russia \\ ${ }^{2}$ Collaborative Laboratory of Cardiovascular Diseases Epidemiology, Novosibirsk, Russia \\ *e-mail: valery.gafarov@gmail.com
}

Key words: NPAS2, gene polymorphism, sleep disorders

Motivation and Aim: To study the prevalence and NPAS2 gene association with sleep disorders as cardiovascular risk factor in male population in Russia/Siberia.

Methods and Algorithms: In 2014-2016 a random representative sample of the male population 25-44 years of one of the districts of Novosibirsk was examined. A random method was used to select 200 men (mean age $=35.5$ years) who underwent psychosocial testing using the C.D. Jenkins scale "4-item Jenkins Sleep Questionnaire". In men, included in the study, the frequency distribution of genotypes rs4851377 of the NPAS2 gene was studied. Approved by Ethical Board. Differences in the frequency distribution of the rs4851377 of the NPAS2 gene between the groups were evaluated by the Chi square test (X2).

Results: Most of men needed 7 hours of sleep per day $-46.7 \%$. The rates of those with an 8-hour sleep were $24.4 \%$. Genotype C/C of gene NPAS2 rs4851377 is more common in those who slept in the day at least 8 hours $(33.3 \%)$ and 9 hours $(33.3 \%)$. Genotype $\mathrm{C} / \mathrm{T}$ and $\mathrm{T} / \mathrm{T}$ were in persons with 7 -hour sleep (50 and $53.3 \%$, respectively). Allele $\mathrm{T}$ carriers in 4.5-fold higher had 6 hours of sleep compared to the $\mathrm{C}$ allele carriers whose sleep was 9 hours. Allele T carriers had lower sleep duration ( 7 instead of 9 hours of sleep) in 4 times compared with allele $\mathrm{C}$.

Conclusion: The findings suggest that site rs4851377 of gene-candidate NPAS2 determines need for sleep in men. 